entropy

ISSN 1099-4300

(C) 2000 by MDPI

www.mdpi.org/entropy/

\title{
Entropy In the Universe: a New Approach
}

\author{
Antonio Alfonso-Faus \\ E.U.I.T. Aeronáutica, Plaza Cardenal Cisneros s/n Madrid 28040, Spain \\ E-mail: aalfonso@euita.upm.es
}

Received: 10 May 2000 / Accepted: 29 July 2000 / Published: 7 September 2000

\begin{abstract}
We propose a new definition of entropy for any mass $\mathrm{m}$, based on gravitation and through the concept of a gravitational cross section. It turns out to be proportional to mass, and therefore extensive, and to the age of the Universe. It is a Machian approach. It is also the number of gravity quanta the mass has emitted through its age. The entropy of the Universe is so determined and the cosmological entropy problem solved.
\end{abstract}

Keywords: entropy, gravity quanta, black holes, Mach, cosmology.

\section{Introduction}

Gravitational radiation (gravitons) is predicted by general relativity. So far no direct detection has been achieved. And this radiation is certainly not responsible for the Newtonian force. One can postulate the existence of gravity quanta responsible for this Newtonian gravitational force. The emission of these gravity quanta can be interpreted as an increase in entropy, and for that one needs a new approach to this concept. Here we give this new definition linked to the concept of a gravitational cross section.

By introducing the concept of a gravitational cross section $\sigma_{\mathrm{g}}$, valid for any object of mass $\mathrm{m}$, one also links the LAB properties with the COSMOLOGICAL ones. By doing so we obtain a Machian result. This has important implications in relation to the long sought connection between quantum mechanics (LAB properties) and general relativity (cosmological parameters).

\section{The Gravitational Cross Section}

To start with we follow a heuristic Newtonian approach to gravitation. We consider the maximum momentum content of a mass $\mathrm{M}$ (energy/ $\mathrm{c}^{2}$ ) to be Mc before it has any given direction (the vector characteristic of momentum), c being the speed of light. For the mass $M$ to have gathered this mo- 
mentum we need a rate of transmission of the order of Mc/t, where $t$ is the age of the Universe. In this heuristic approach we assume that the maximum momentum content of a mass $\mathrm{M}$ has been built during the age of the Universe. The ratio Mc/t is a force and we assume here that it refers to the maximum gravitational interaction a body of mass $\mathrm{M}$ can have on its surroundings. Implied here is the view that the maximum momentum content $\mathrm{Mc}$ is due to the gravitational interaction on $\mathrm{M}$ due to the rest of the Universe during its age, a Machian approach. We will now take into account the geometry. We now define the gravitational cross section in terms of the effectiveness in momentum transfer. This is assumed to occur by means of the absorption of the gravity quanta, which need to have their momentum opposite to their velocity. The maximum gravitational interaction from a source $M$ would be spread over an area $4 \pi r^{2}$, the surface of the sphere of radius $r$ centered on M. Considering a test mass $m$ on this surface, the gravitational interaction of $\mathrm{M}$ on $\mathrm{m}$ would be proportional to the ratio $\sigma_{\mathrm{g}} / 4 \pi \mathrm{r}^{2}$, where $\sigma_{\mathrm{g}}$ is the gravitational cross section of the mass $\mathrm{m}$, the area for gravitational interaction from any mass M. Hence, Newton's gravitational force between $\mathrm{M}$ and $\mathrm{m}$ can be written, as far as the scalar value is concerned, as

$$
\frac{G M m}{r^{2}} \approx \frac{M c}{t} \frac{\sigma_{g}}{4 \pi r^{2}}
$$

From here one gets

$$
\sigma_{g} \approx 4 \pi \frac{G m t}{c}
$$

The size of the Universe R can be taken to be of the order of ct, hence one has from (2)

$$
\sigma_{g} \approx 4 \pi \frac{G m}{c^{2}} R
$$

Here we have a Machian definition of the gravitational cross section for a mass $\mathrm{m}$. It is given by the product of its gravitational radius $\mathrm{Gm} / \mathrm{c}^{2}$ times the size of the Universe R. Since the size of the Universe $\mathrm{R}$ is of the order of its gravitational radius $\mathrm{GM}_{\mathrm{u}} / \mathrm{c}^{2}$, where $\mathrm{M}_{\mathrm{u}}$ is he mass of the Universe, one has that the square root of the gravitational cross section of $\mathrm{m}$ (its "gravitational size" $\lambda_{\mathrm{g}}$ as presented by Alfonso-Faus [1]) is of the order of the geometrical mean of two gravitational radii: the gravitational radius of the mass $m$ and the gravitational radius of he Universe:

$$
\lambda_{g}=\sqrt{\sigma_{g}} \approx \sqrt{4 \pi \frac{G m}{c^{2}} \frac{G M_{u}}{c^{2}}}
$$

Let us check this value with some examples. In the case of a proton one has a gravitational radius of about $10^{-52} \mathrm{~cm}$, and since the size of the Universe is about $10^{28} \mathrm{~cm}$ one gets the geometrical mean of 
$10^{-12} \mathrm{~cm}$, which is about the Compton wavelength $\lambda_{\mathrm{c}}=\hbar / \mathrm{m}_{\mathrm{p}} \mathrm{c}$ of the proton with mass $\mathrm{m}_{\mathrm{p}}$, where $\hbar$ is Planck's constant. In the case of the sun one has for the gravitational size $10^{16} \mathrm{~cm}$, which is about the solar system size. For a typical galaxy we get a gravitational size of $10^{22} \mathrm{~cm}$, which is about its actual size. Hence we have a physical meaning for the gravitational size of any mass $\mathrm{m}$ : it turns out to be the size of its gravitational influence, the actual size for the proton, the galaxies and the Universe.

\section{The Gravitational Size and the Expansion of the Universe}

The gravitational size $\lambda_{\mathrm{g}}$, being the square root of the gravitational cross section given by (2), is proportional to $\mathrm{t}^{1 / 2}$. A dimensional analysis of the Einstein cosmological equations (to be presented next September at the Spanish annual E.R.E. relativity meeting) gives that the factor $\mathrm{Gm} / \mathrm{c}$ in (2) is a real constant (in the sense that it is not affected by the expansion of the Universe), and that the general solution for the cosmological scale factor $a$ is $a(\mathrm{t})=\mathrm{t}^{1 / 2}$ in a certain system of units. Hence we get the result that the gravitational sizes expand as the Universe does. They are proportional to the cosmological scale factor.

\section{A New Definition for Entropy}

There is another important interpretation for the meaning of the gravitational cross section. In units of the square of Planck's length $1_{*}=\left(\mathrm{G} \hbar / \mathrm{c}^{3}\right)^{1 / 2}$ one can define a gravitational entropy, $\mathrm{S}_{\mathrm{mg}} / \mathrm{k}$ for any mass $\mathrm{m}$, where $\mathrm{k}$ is the Boltzmann constant Alfonso-Faus [1] (note that in this paper $\mathrm{A}_{\mathrm{e}}=\sigma_{\mathrm{g}}$ here):

$$
\frac{\sigma_{g}}{l_{*}^{2}}=\frac{S_{m g}}{k} \approx 4 \pi \frac{G m}{c^{2}} c t \frac{1}{l_{*}^{2}}
$$

This definition has to be compared with the definition of entropy given by Bekenstein [2] and Hawking [3] for a black hole. These authors definition of entropy as being proportional to the area of the event horizon, and hence to the square of the mass, gives a non-extensive property for the entropy. In our definition of gravitational entropy the extensive property is preserved: if one doubles the mass the entropy doubles too. And it increases linearly with time, which preserves the second law of thermodynamics. Entropy as an extensive property and increasing with time are well known conditions from classical thermodynamics. Also these authors use Planck's length as a reference for the horizon area. If one uses Planck's length $1_{*}$ as the unit reference one gets for our definition of entropy:

$$
\frac{\sigma_{g}}{l_{*}^{2}} \approx \frac{S_{m g}}{k} \approx 4 \pi \frac{m c^{2}}{\hbar} t
$$

This is a very important result. As seen from the LAB reference, the atomic clocks have a tic proportional to $\hbar / \mathrm{mc}^{2}$. Hence the entropy so defined is about the number of time intervals $\hbar / \mathrm{mc}^{2}$ contained in the age of the Universe t. It is the number of "tics" the particle has undergone during its age. It can be interpreted as the number of gravity quanta emitted (see Alfonso-Faus [1]) during the age of the Universe. For an elementary particle this entropy today is about $10^{41}$. For the Universe this entropy is 
about $10^{121}$. Under this view the contribution to the entropy of the Universe given by the background radiation is now negligible. The linear dependence of entropy with time explains why there is so much entropy in the Universe today: this is just because the Universe is old. The problem to explain such high entropy in the Universe is thus solved.

The definition of entropy for any mass $m$ given in this work is just the number of gravity quanta emitted by this mass during the age of the Universe. The mass $\mathrm{m}_{1}$ of a gravity quantum is given by Alfonso-Faus [1]

$$
m_{1}=\frac{\hbar}{c^{2} t}
$$

The entropy is then the number of these gravity quanta in the mass $\mathrm{m}$ :

$$
\frac{S_{m g}}{k} \approx \frac{m}{m_{1}}=\frac{m c^{2}}{\hbar} t
$$

which coincides with the relation given in (6). Hence the entropy of any mass $m$ can be defined as the gravitational cross section of this mass, in units of Planck's length squared, or equivalently the number of "tics" that the mass has undergone during the age of the Universe (i.e. the number of emitted gravity quanta).

\section{Conclusion}

The entropy of any mass $\mathrm{m}$, including black holes, may be defined in terms of a gravitational cross section that turns out to be proportional to the mass $m$ and the age of the Universe $t$. This gives a Machian approach to entropy. The gravitational size related to this gravitational cross section expands as the Universe does. This entropy has an extensive property and increases linearly with time. It is in fact the number of gravity quanta that each mass has emitted during its age. The gravity quanta present in the Universe are just its entropy now, about $10^{121}$ in units of Boltzmann's constant.

\section{References and Notes}

1. Alfonso-Faus, A. Physics Essays 2000, Vol. 12 N4, December and preprint arXiv:gr-qc/0002065 18 Feb. 2000.

2. Bekenstein, J. Phys. Rev. 1972, D7, 2333-2346.

3. Hawking, S. W. Commun. Math. Phys. 1975, 43, 199-220.

(C) 2000 by MDPI. All rights reserved. 